\title{
THE ORIGIN OF SO 2 AFTERGLOWS AS REVEALED BY MAGNETIC FIELD EFFECTS
}

\author{
HARUO ABE \\ Molecular Photochemistry Laboratory, The Institute of Physical and Chemical \\ Research (RIKEN). Wako, Saitama 351-01, Japan
}

(Received 14 March, 1993)

The chemiluminescence intensity in the low pressure diffusion flame of the $\mathrm{CS}_{2}+\mathrm{O} / \mathrm{N}_{2}$ system was found to be considerably affected by external magnetic fields. The emitter in the flame was identified as the main emitter in the $\mathrm{SO}_{2}$ afterglow. The measurements of the field strength dependence, collisional effect, and spectral distribution of the magnetic field effect revealed the major emitter as the $\mathrm{SO}_{2}$ in the $\widetilde{\mathrm{C}}$ state. External magnetic fields were also found to quench fluorescence remarkably emitted from the vibronic levels just below the predissociation threshold in the $\widetilde{\mathrm{C}}$ state. From the observed vibrational-level, field-strength, and pressure dependences of the magnetic quenching, it became evident that the major emitter of chemiluminescence in the flame could be assigned to the $\mathrm{SO}_{2}$ molecule in the vibronic levels located at about $800 \mathrm{~cm}^{-1}$ below the predissociation threshold in the $\widetilde{\mathrm{C}}$ state

KEY WORDS: Chemiluminescence, $\mathrm{SO}_{2}$ afterglow, magnetic field effect, fluorescence excitation spectra, predissociation, recombination reaction.

\section{INTRODUCTION}

$\mathrm{SO}_{2}$ is one of the key molecules in atmospheric and combustion reactions. It is Gaydon ${ }^{1}$ who first recorded the spectrum of $\mathrm{SO}_{2}$ afterglow using a discharged flow of pure $\mathrm{SO}_{2}$ at $0.1-5$ Torr. He indicated that the emission source was electronically excited $\mathrm{SO}_{2}\left(\mathrm{SO}_{2}{ }^{*}\right)$, which was produced by the recombination of $\mathrm{SO}$ and $\mathrm{O}$. Herman et al. ${ }^{2}$ confirmed this by showing that the short wavelength limit of the chemiluminescence emission corresponds in the energy to the onset of continuous absorption by $\mathrm{SO}_{2}$, which is now known ${ }^{3,4}$ to be due to the predissociation threshold of $\mathrm{SO}_{2} \rightarrow$ $\mathrm{SO}\left(\mathrm{X}^{3} \Sigma^{-}\right)+\mathrm{O}\left({ }^{3} \mathrm{P}\right)$. Using a fast flowing afterglow apparatus, Halstead and Thrush ${ }^{5}$ showed that $\mathrm{SO}_{2}{ }^{*}$ is formed by a three-body process in the afterglow not by a two-body radiative association of $\mathrm{SO}$ and $\mathrm{O}$. Main process is

$$
\mathrm{SO}+\mathrm{O}+\mathrm{M} \rightarrow \mathrm{SO}_{2}^{*}+\mathrm{M}
$$

with a rate constant of $(3.2 \pm 0.4) \times 10^{17} \mathrm{~cm}^{6} \mathrm{~mole}^{-2} \mathrm{~s}^{-1}$ at $300 \mathrm{~K}$ for $\mathrm{M}=\mathrm{Ar}^{5}$

The reverse reaction of (1) without $\mathrm{M}$ is the dissociation of $\mathrm{SO}_{2}$ as suggested above. The onset of the corresponding predissociation of $\mathrm{SO}_{2}$ in the third excited singlet state $\widetilde{C}{ }^{1} \mathrm{~B}_{2}$ was found by $\mathrm{Okabe}^{3}$ and recently Ebata et al. ${ }^{4}$ determined the threshold energy to be $45400 \mathrm{~cm}^{-1}$ from the sudden decreases of fluorescence yields 
and lifetimes. In our previous paper, ${ }^{6}$ we reported that fluorescence from the vibronic levels located just below the threshold is quenched remarkably by external magnetic fields below $12 \mathrm{kG}$ in a collision free condition. From the field strength dependence, the magnetic quenching could be interpreted in terms of the radiationless transition theory in a magnetic field. ${ }^{7,8}$ The magnetic quenching rate increases rapidly with increasing the vibrational energy of the emitting level until the threshold and it drops suddenly above the threshold. This energy dependence of the magnetic quenching indicates that the mechanism of the magnetic sensitive radiationless transition would be closely relevant to that of the predissociation.

In the present study, we have measured external magnetic field effect (MFE) on the emission intensity of the $\mathrm{SO}_{2}$ afterglow paying special attention to its spectral distribution and its total pressure dependence in the reaction region. We have also measured the MFE on the laser-induced fluorescence intensities and its decay profiles emitted from the vibronic levels of the $\mathrm{SO}_{2} \mathrm{C}$ state at various pressures of $\mathrm{N}_{2}$, which served as a collision partner. The main purpose of the present paper is to clarify the link between the emitting species in the $\mathrm{SO}_{2}$ afterglow and the vibronic levels in the $\mathrm{SO}_{2} \mathrm{C}$ state below the predissociation threshold from a viewpoint of the MFEs on the emissions from them.

\section{EXPERIMENTAL}

The chemiluminescence spectra, fluorescence excitation spectra, and fluorescence decays were measured under various magnetic fields up to $12 \mathrm{kG}$ with the same apparatus as reported elsewhere. ${ }^{6,9,10}$ In stead of the cross-diffusion setup, we applied a burner-type setup to forming the low pressure diffusion flame of $\mathrm{CS}_{2}+\mathrm{O} / \mathrm{N}_{2}$ in this study, as shown in Figure 1. By restricting the reaction region to smaller volume, this setup gave brighter chemiluminescence than the cross-type one. Oxygen atoms were produced by adding $\mathrm{NO}$ to a microwave discharged $\mathrm{N}_{2}$ stream. This is a wellestablished method for obtaining atomic oxygen without chemically active molecular oxygen., ${ }^{9,11,12}$ In our flow system, the NO titration was carried out at a point $18 \mathrm{~cm}$ downstream from the discharge region. A equimoler point of $[\mathrm{NO}]=[\mathrm{N}]_{0}\left([\mathrm{~N}]_{0}\right.$ denotes the flow rate of $\mathrm{N}$ atom at the titration point) was reached at $[\mathrm{NO}]=4.22$ $\mu \mathrm{mole} / \mathrm{s}$ for the $\left[\mathrm{N}_{2}\right]=273 \mu \mathrm{mole} / \mathrm{s} .^{9}$ Therefore the flow rate of $\mathrm{O}$ atom at the point was considered to be 4.22 Omole/s. All results described below were obtained under this condition. $\mathrm{CS}_{2}$ vapor encountered with the $\mathrm{O} / \mathrm{N}_{2}$ flow and as a result, the chemiluminescent species in the diffusion flame were produced near the center of an octagonal duralmin chamber placed between the poles of an electromagnet. Chemiluminescence was dispersed by a monochromator and detected by a photomultiplier. Photon signals were processed by a photon counter system. ${ }^{9}$

For the measurements of the laser excitation spectra and the fluorescence time profiles, the second harmonic of a $\mathrm{XeCl}$ excimer laser pumped dye laser excited the $\mathrm{SO}_{2}$ vapor, which was effused continuously into the chamber through a tube of $4 \mathrm{~mm}$ diameter. The laser-induced fluorescence was detected by a photomultiplier through a monochromator. The pulsed signals were transferred to a boxcar integrator 


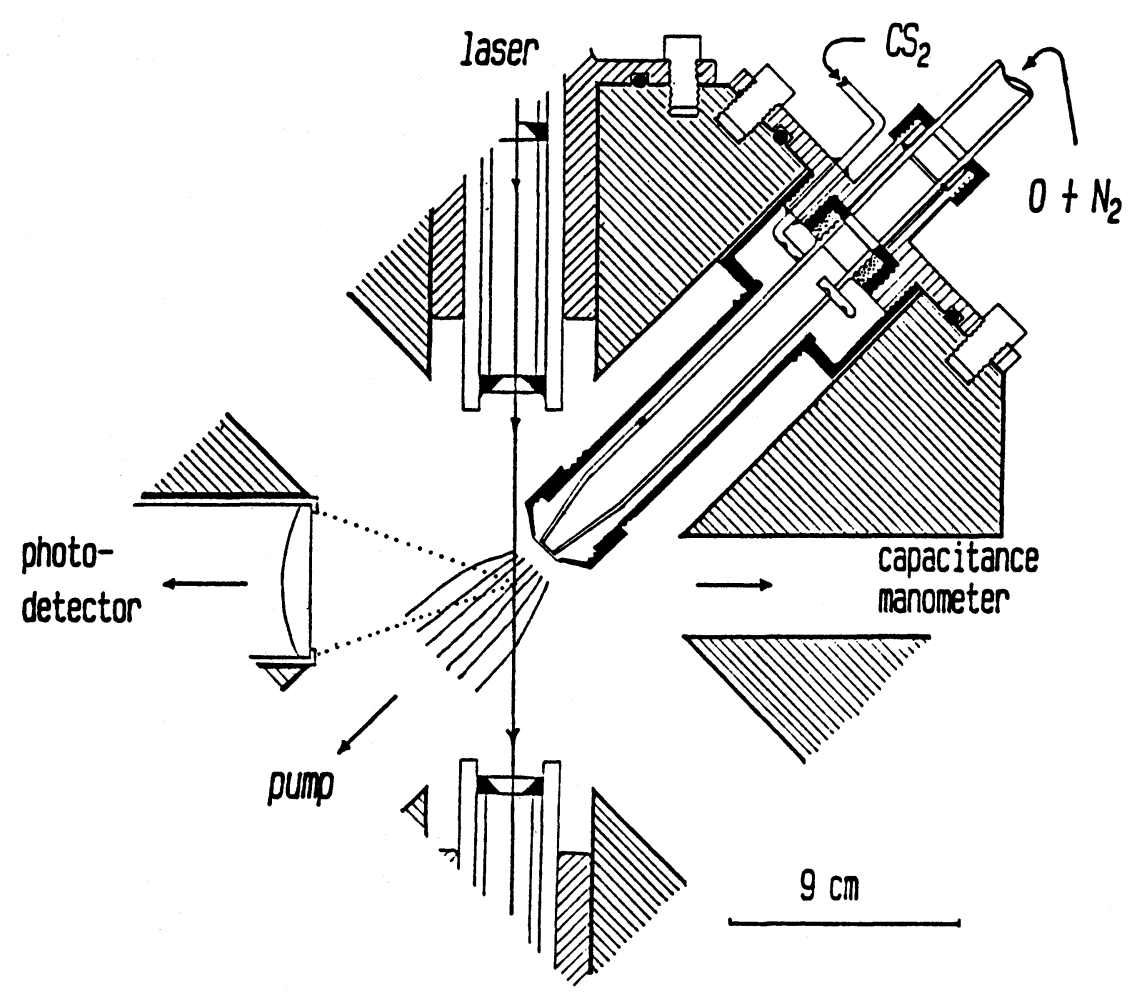

Figure 1 Schematic of a burner-type reaction setup to form the low pressure diffusion flame of the $\mathrm{CS}_{2}+\mathrm{O} / \mathrm{N}_{2}$ system. The octagonal chamber is placed between the poles of $9 \mathrm{~cm}$ diameter of an electromagnet.

or a digital oscilloscope. A personal computer was used for data aquisition, accumulation, and analysis via a GPIB interface. ${ }^{6,9,13}$

$\mathrm{N}_{2}(>99.99 \%)$, $\mathrm{NO}(>99.0 \%)$, and $\mathrm{SO}_{2}(>99.9 \%)$ were used without further purification. Spectrograde $\mathrm{CS}_{2}$ (Merk) was degassed by repeated freeze-pump-thaw cycles. The flow rates of all sample vapors were controlled by mass-flow controllers. As a result, the partial pressures (relative concentrations) of the component gases and the total pressure could be set and kept within the errors of $\pm 0.5 \% .^{9}$ Pressures in the chamber were monitored by a capacitance manometer.

The detection system and the microwave discharge region were sufficiently shielded against the leakage of magnetic fields. 9

\section{RESULTS AND DISCUSSION}

\section{Magnetic Field Effect on Chemiluminescence Intensity}

Figure 2 shows chemiluminescence spectra of $\mathrm{CS}_{2}+\mathrm{O} / \mathrm{N}_{2}$ diffusion flames at $\mathrm{H}$ $=0$ and $12 \mathrm{kG}$. Comparing with the spectrum of the $\mathrm{SO}_{2}$ afterglow obtained by 


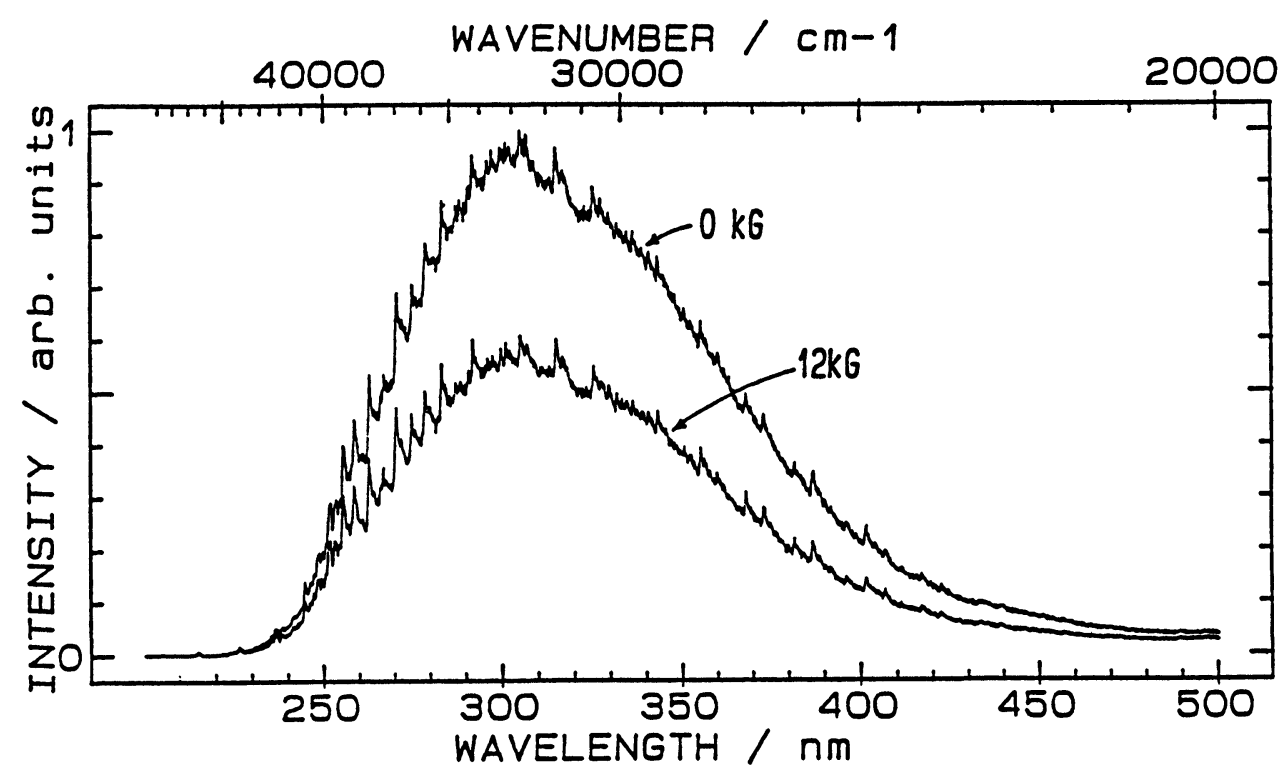

Figure 2 Chemiluminescence spectra from the low pressure diffusion flames of the $\mathrm{CS}_{2}+\mathrm{O} / \mathrm{N}_{2}$ system under the presence and absence of a magnetic field of $12 \mathrm{kG}$ at the pressure of 1.464 Torr. The flow rates of $\mathrm{NO}, \mathrm{CS}_{2}$ and $\mathrm{N}_{2}$ are $4.22,1.09$ and $273 \mu \mathrm{mole} / \mathrm{s}$, respectively. The spectral sensitivity of the detection system is not corrected.

Halstead and Thrush, ${ }^{5}$ our spectra are found to correspond to the $\alpha$ system of $\mathrm{SO}_{2}$, which has been known to correlate with the $\mathrm{SO}_{2}$ predissociation limit. The ${ }^{1} \mathrm{~B}$ and ${ }^{3} \mathrm{~B}_{1}$ emissions ${ }^{5}$ were not observed in our spectra. This is probably due to the absence of molecular oxygen in our system. The reactions in the flame that produce the emission $\operatorname{are}^{5}$

$$
\begin{gathered}
\mathrm{CS}_{2}+\mathrm{O} \rightarrow \mathrm{CS}+\mathrm{SO}, \\
\mathrm{SO}+\mathrm{O}+\mathrm{N}_{2} \rightarrow \mathrm{SO}_{2}{ }^{*}+\mathrm{N}_{2},
\end{gathered}
$$

and

$$
\mathrm{SO}_{2} * \rightarrow \mathrm{SO}_{2}+\mathrm{h} v \text {. }
$$

Recent study ${ }^{14}$ using time-resolved infrared laser absorption shows that the reaction (2) is the main branching channel $(\sim 90 \%)$ of $\mathrm{O}\left({ }^{3} \mathrm{P}\right)+\mathrm{CS}_{2}$ reaction. The reaction (3) is the same as (1) with $M=N_{2}$. The red-degraded sharp band structure seen in Figure 2 is not due to (4) but can be assigned to the $\mathrm{SO}\left(\mathrm{B}^{3} \Sigma^{-} \mathrm{v}^{\prime}=0-3 \rightarrow \mathrm{X}^{3} \Sigma^{-}\right.$ $\left.\mathrm{v}^{\prime \prime}=0-17\right)$ bands. ${ }^{15,16}$ This $\mathrm{SO}(\mathrm{B}-\mathrm{X})$ emission was observed with relatively stronger intensity at the higher pressure, indicating that the $\mathrm{SO}(\mathrm{B})$ is formed by a secondary reaction channel.

The key observation in the present study is the external magnetic field quenching of the $\mathrm{SO}_{2}{ }^{*}$ emission as seen in Figure 2. A magnetic field $(\mathrm{H})$ of $12 \mathrm{kG}$ decreases the emission intensity by more than $40 \%$ of that at $\mathrm{H}=0 \mathrm{kG}$. In contrast, the 
$\mathrm{SO}(\mathrm{B}-\mathrm{X})$ emission is not affected by the field. The $\left[\mathrm{CS}_{2}\right]$ dependence of the emission intensity is shown in Figure 3a. As established by Halstead and Thrush, ${ }^{5}$ the emission intensity $\left(\mathrm{I}_{\mathrm{em}}\right)$ is proportional to $[\mathrm{O}]$ and $[\mathrm{SO}]$ at the pressures between $0.23-5$ Torr as

$$
\mathrm{I}_{\mathrm{em}} \propto[\mathrm{O}][\mathrm{SO}] .
$$

Assuming that the reactions (2) and (3) occur sequentially at $\left[\mathrm{CS}_{2}\right]<[\mathrm{O}]$, the equation (5) is approximately to be

$$
\mathrm{I}_{\mathrm{em}} \propto\left\{[0]_{0}-\left[\mathrm{CS}_{2}\right]\right\}\left[\mathrm{CS}_{2}\right]=-\left\{\left[\mathrm{CS}_{2}\right]-[\mathrm{O}]_{0} / 2\right\}^{2}+[\mathrm{O}]_{0}^{2 / 4}
$$

where $[\mathrm{O}]_{0}$ is the initial flow rate of atomic oxygen at the reaction region. As can be seen in Figure 3a, the quadratic intensity dependence (6) on $\left[\mathrm{CS}_{2}\right]$ fairly holds in

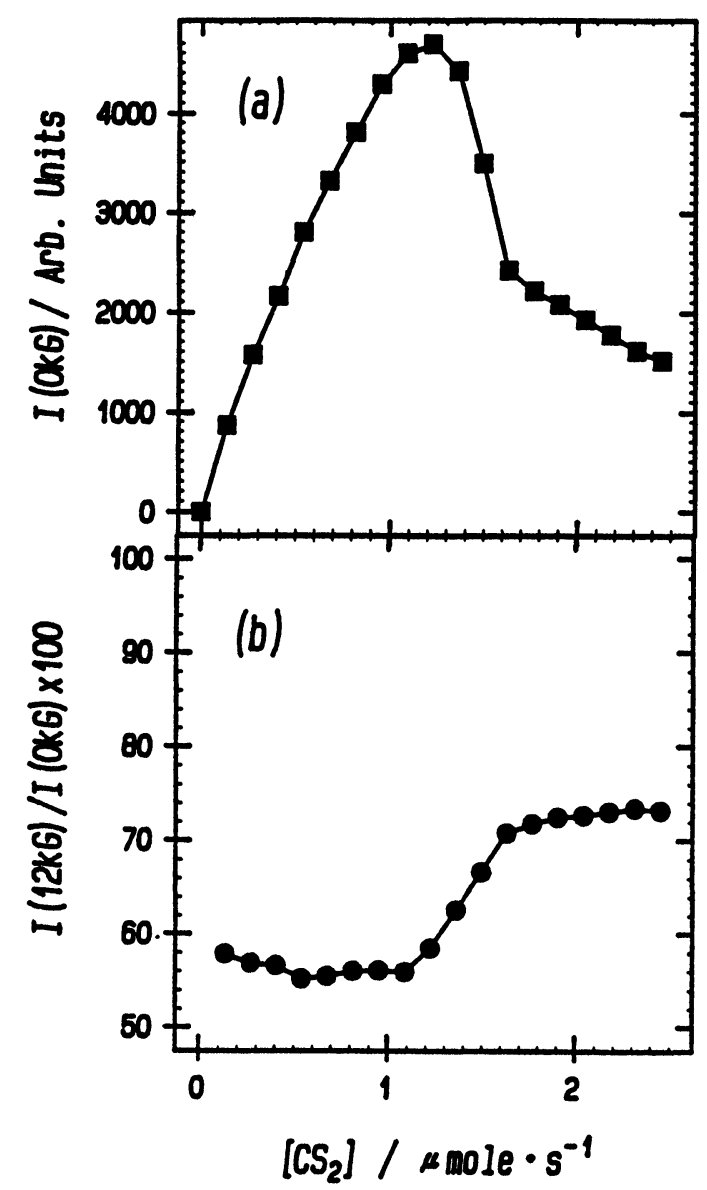

Figure $3\left[\mathrm{CS}_{2}\right]$ dependences of (a) chemiluminescence intensity and (b) its magnetic field quenching at $\mathrm{H}=12 \mathrm{kG}$. The pressure varies from 2.032 to 2.058 Torr with the addition of $\mathrm{CS}_{2}$. The emission is monitored at $313 \pm 0.5 \mathrm{~nm}$. 
the region of $\left[\mathrm{CS}_{2}\right]<1.2 \mu \mathrm{mole} / \mathrm{s}$. And in this region, the efficiency of the magnetic quenching is almost constant as shown in Figure $3 \mathrm{~b}$. From the position of the intensity peak and the equation (6), $[\mathrm{O}]_{0}$ is estimated to be $2.4 \mu \mathrm{mole} / \mathrm{s}$, which is a result from the reaction with the wall of the flow tube during the $28 \mathrm{~cm}$ flow from the NO titration point to the reaction region. In the region of $\left[\mathrm{CS}_{2}\right]>[\mathrm{O}]_{0} / 2$, the stoichiometry of the reactions (2) and (3) does not hold and $\mathrm{I}_{\mathrm{em}}$ follows an irregular dependence of $\left[\mathrm{CS}_{2}\right]$. Interestingly, the magnetic quenching shows a parallel dependence to $\mathrm{I}_{\mathrm{em}}$ in this region. In the following measurements, we fixed $\left[\mathrm{CS}_{2}\right]$ at $1.09 \mu \mathrm{mole} / \mathrm{s}$, where the emission intensity is near the peak and the magnetic quenching is in the constant region.

\section{Pressure Dependence of MFE on Chemiluminescence Intensity}

By adjusting the valve located between the reaction chamber and the pumping station, we measured the effect of the total pressure $(\mathrm{P})$ on the $\mathrm{SO}_{2}{ }^{*}$ emission intensity and on the efficiency of the magnetic field quenching with the constant flow rates of $\mathrm{N}_{2}, \mathrm{NO}$ and $\mathrm{CS}_{2}$. The results are shown in Figure 4. The intensity dependence has two maxima at $\mathrm{P}=2$ Torr and at $\mathrm{P}=6$ Torr. At the intensity maximum at the lower pressure, the magnetic quenching has a peak in the efficiency. The field strength dependences of the MFE were measured for five pressure points. The results are plotted in Figure 5. In the low pressure ( $<\sim 1$ Torr) region, the emission intensity increases with increasing $\mathrm{H}$ until $1 \mathrm{kG}$ and decreases at the higher fields. As the pressure increases, the magnetic enhancements at low fields are diminished and the magnetic quenchings become predominant. Note that the magnetic field quenchings are not saturated even at the highest field obtained, except for those at $\mathrm{P}=0.493$ Torr.

\section{Spectral Distribution of MFE on Chemiluminescence Intensity}

In order to clarify somewhat peculiar field dependence of the MFE upon the total pressure, we measured its spectral distributions at $P=0.493$ Torr. As shown in Figure 6, there should be (at least) two emitting species in the flame. The major species dominates the shape of the emission spectra and its emission is quenched by magnetic fields. The minor one has its spectral distribution in the higher energy region than that of the major one and its emission is enhanced greatly by relatively low fields. At $\mathrm{H}=1 \mathrm{kG}$, the enhancement of the minor emission surpasses the quenching of the major emission. As seen in Figure 6b, even at $12 \mathrm{kG}$ the magnetic quenching of the major emission is partly blurred in the short wavelength region $(<250 \mathrm{~nm})$ where the minor emission contribution is large. Thus the minor emission should be enhanced rapidly by low fields and this enhancement would slow down or possibly be saturated at higher fields.

The major species can be reasonably attributed to $\mathrm{SO}_{2}{ }^{*}$ as discussed above. The results shown in Figures 4 and 5 were obtained by monitoring the emissions at 313 $\pm 0.5 \mathrm{~nm}$ and can be considered to be those for the major species except for those in the low pressure region of Figure 5. We are mainly concerned with the major species i.e. $\mathrm{SO}_{2} *$ hereafter. 


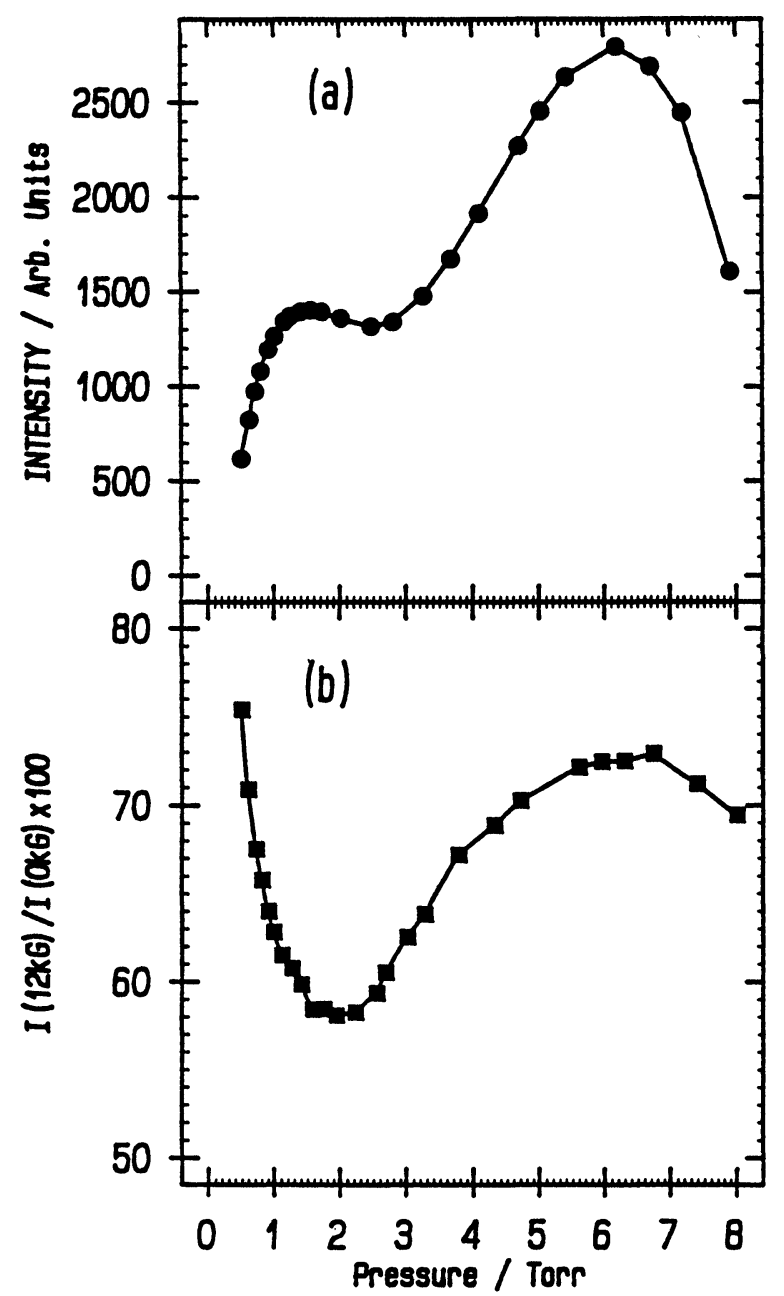

Figure 4 Total pressure dependences of (a) chemiluminescence intensity and (b) its magnetic field quenching at $\mathrm{H}=12 \mathrm{kG}$. The emission is monitored at $313 \pm 0.5 \mathrm{~nm}$. Other conditions are the same as Figure 2.

\section{MFE on Fluorescence Intensity Emitted from $\mathrm{SO}_{2} \widetilde{C}$ State}

Chemiluminescence observed in our system is attributed to the emission from the $\mathrm{SO}_{2} \alpha$ state of references 5 . The $\alpha$ state $^{17}$ is now known to be the $\mathrm{SO}_{2} \mathrm{C}^{1} \mathrm{~B}_{2}$ state. ${ }^{18,19}$ Thus we have started to observe the MFE on the fluorescence intensities from the vibronic levels of the $\widetilde{C}$ state in a collision free condition. We have briefly reported the results of the measurements in our previous paper. ${ }^{6}$ The most prominent result is displayed in Figure 7 for the fluorescence excitation spectra of the head portion of the $\left(\mathrm{v}_{1} \mathrm{v}_{2} \mathrm{v}_{3}\right)=(132)$ vibronic band under various magnetic fields. This level is located just below the predissociation threshold. ${ }^{4}$ As seen in the Figure 7, more than 


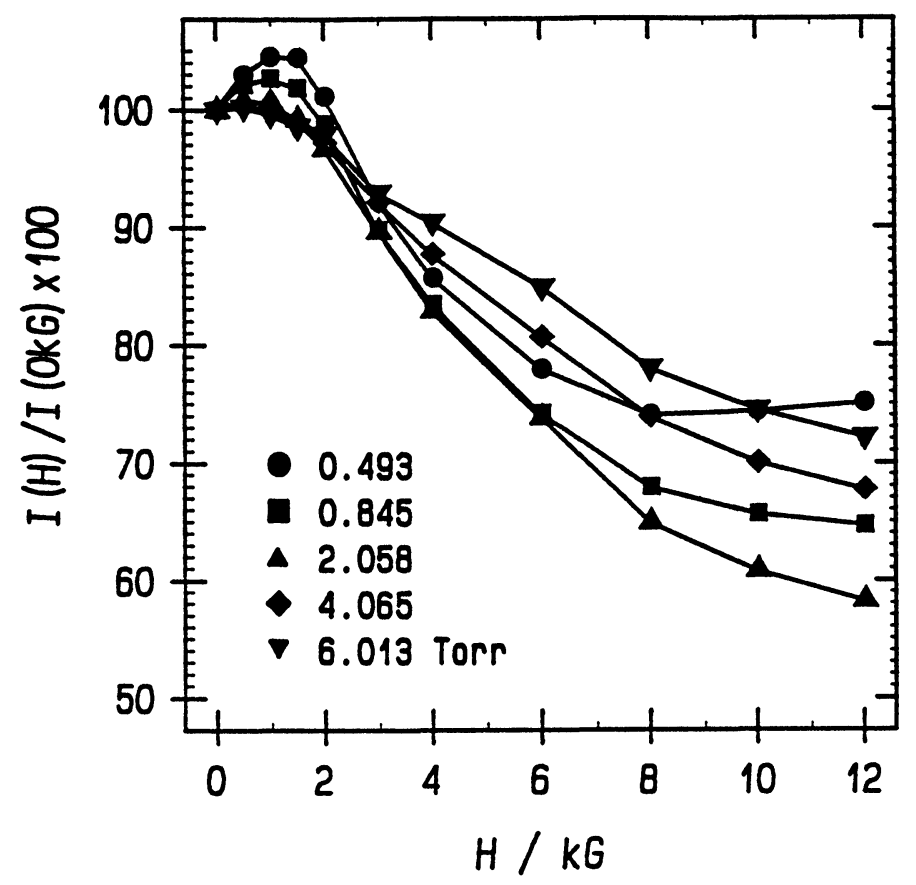

Figure 5 Field strength dependences of the magnetic quenchings at various pressures. The emission is monitored at $313 \pm 0.5 \mathrm{~nm}$. Other conditions are the same as Figure 2 .

$60 \%$ of fluorescence at $\mathrm{H}=0 \mathrm{kG}$ is quenched by a magnetic field of $12 \mathrm{kG}$. Since no Zeeman broadening is noticeable in our resolution $\left(\sim 0.2 \mathrm{~cm}^{-1}\right)$ and since the magnetic quenching is not homogeneous for the peaks of the rotational structure, we measured the field dependences of the quenchings for arbitrarily selected four peaks a-d shown in Figure 7 as the representatives of the band. The results are plotted in Figure 8. The magnetic quenching occurs rapidly at low fields and at high fields the slope of the quenching efficiency becomes gentle but shows no saturation.

In our previous paper, ${ }^{6}$ we demonstrated that the field dependence of the quenching efficiency as shown above can be interpreted in terms of the radiationless transition theory in a magnetic field. ${ }^{78}$ The direct mechanism (DM) is the field-induced coupling between the prepared level and an adjacent quasicontinuum of dark levels by the Zeeman term. This coupling accelerates the radiationless transition, giving the rate constant as

$$
\mathrm{K}_{\mathrm{nr}}^{\mathrm{DM}}(\mathrm{H})=\alpha \mathrm{H}^{2},
$$

where $\alpha$ is the proportionality constant given by the matrix element of the Zeeman hamiltonian. The typical examples for the DM are the MFEs on the $\mathrm{I}_{2}{ }^{*}$ predissociation ${ }^{20}$ and on the fluorescence yield of the $\mathrm{CS}_{2} \mathrm{~V}^{1} \mathrm{~B}_{2}$ state. ${ }^{21}$ 


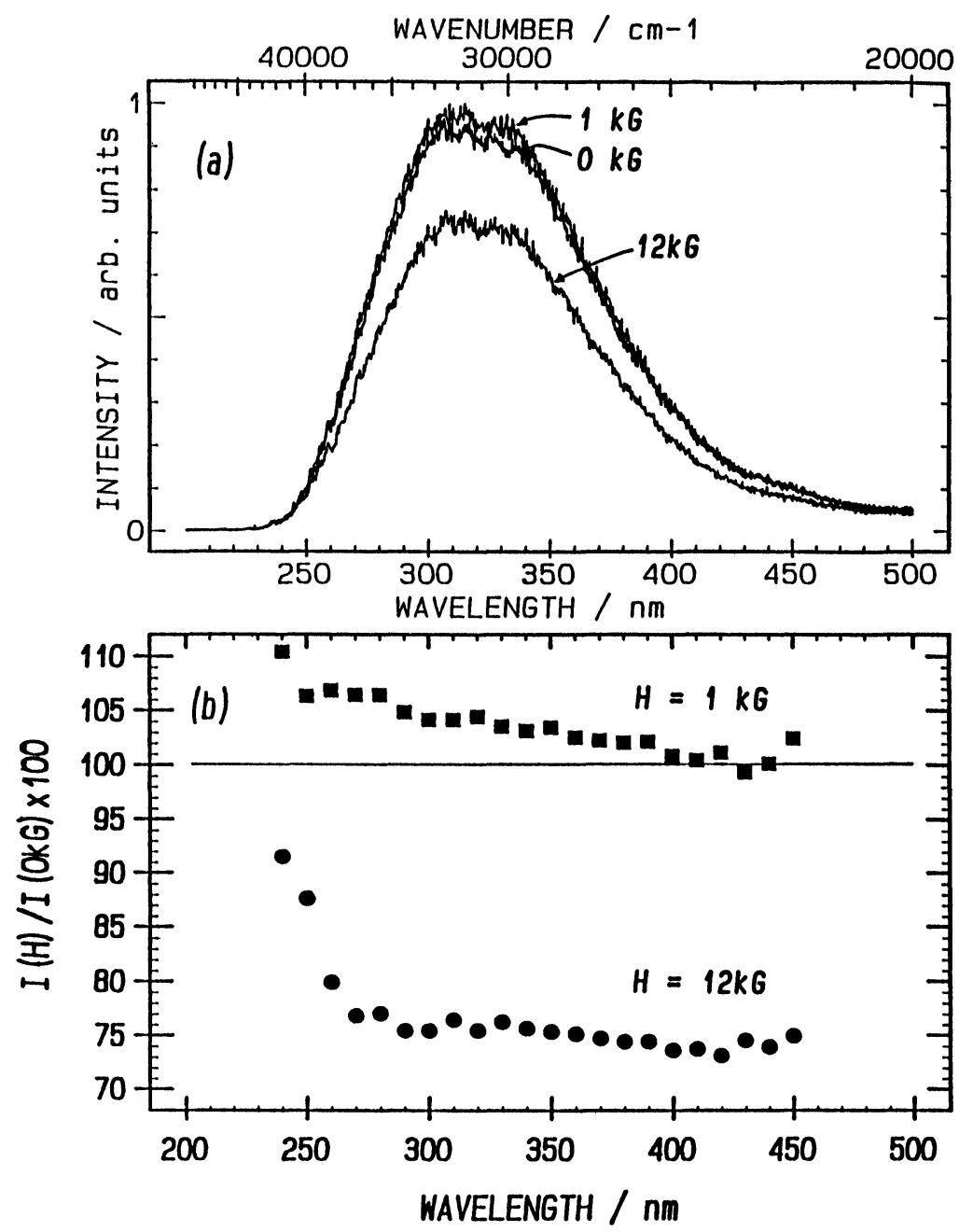

Figure 6 (a) Chemiluminescence spectra at $\mathrm{H}=0,1$ and $12 \mathrm{kG}$ and (b) magnetic field effects on the intensities. Total pressure is 0.493 Torr. Other conditions are the same as Figure 2.

The indirect mechanism (IM) of the magnetic quenching arises, in most cases, from the spin decoupling induced by a magnetic field in the triplet levels with which the photoexcited singlet level interacts through the spin-orbit interaction. In this mechanism, the field-induced nonradiative rate is given by

$$
\mathrm{k}_{\mathrm{nr}}^{\mathrm{IM}}(\mathrm{H})=\beta \mathrm{H}^{2}
$$

in low fields and in high fields in which the spin decoupling is completed, the rate becomes constant. This saturation behavior at relatively low fields can be readily found experimentally and gives clear evidences of the IM for the magnetic quenchings, for examples, in the $S_{1}$ states of aza-benzenes ${ }^{22,23}$ and acetylene. ${ }^{13}$ 


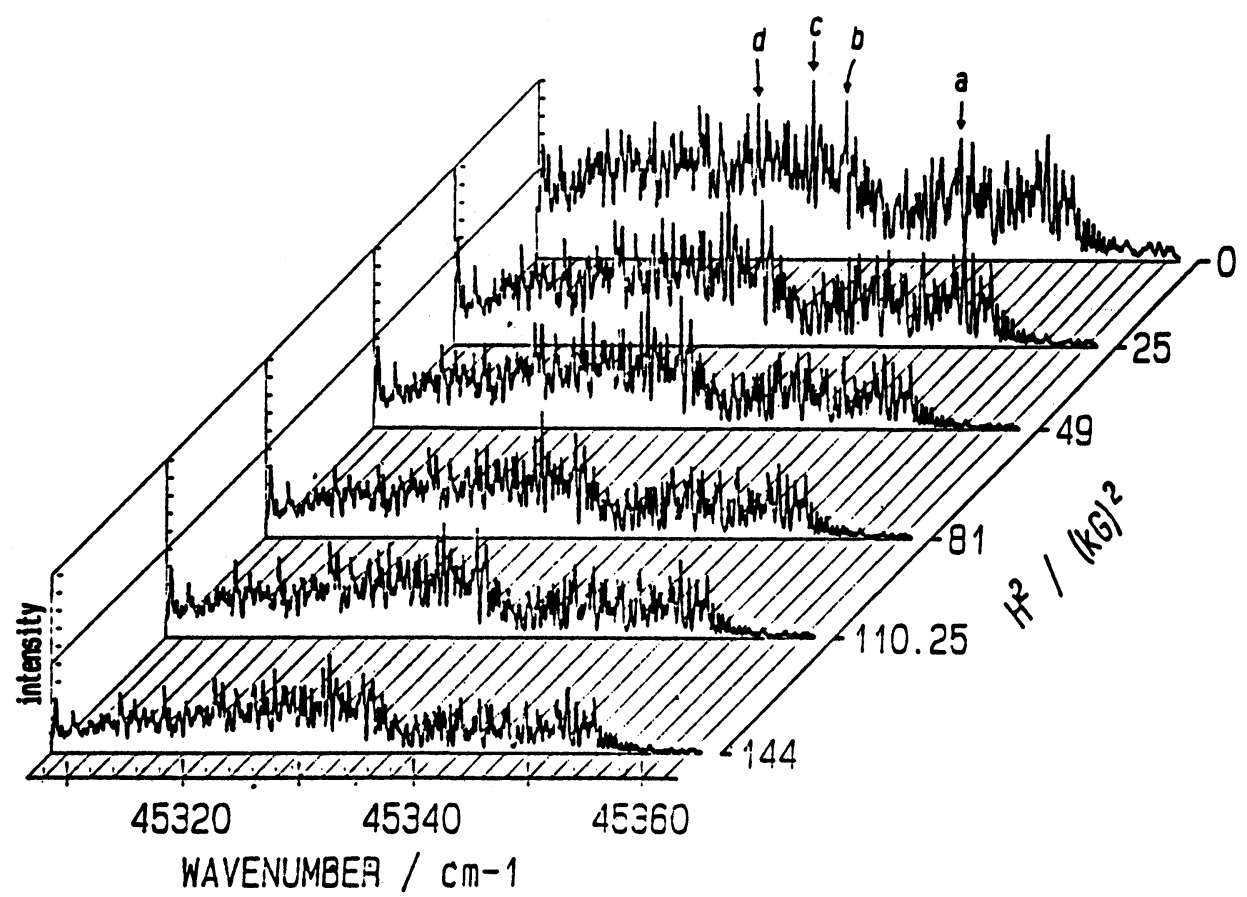

Figure 7 Fluorescence excitation spectra observed around the head of the (132) band of the $\mathrm{SO}_{2} \widetilde{\mathrm{C}}{ }^{1} \mathrm{~B}_{2}$ state at various magnetic fields. The pressure of $\mathrm{SO}_{2}$ is 0.006 Torr, which ensures a collision free condition.

Our results shown in Figure 8 were found to arise from a hybrid of the DM and IM. ${ }^{6}$ The spin decoupling of the IM is completed at $\sim 6 \mathrm{kG}$ and the quenching ratios ${ }^{6-8}$ are proportional to the square of the field strength in the high fields. It is worth noting that the MFEs of the $\mathrm{SO}_{2} *$ chemiluminescence presented in Figure 5 show similar behaviors in the field dependence to those in Figure 8, especially in the high field region, although the efficiencies of the quenching are small. This fact presents another evidence to indicate that the main emitter in the $\mathrm{SO}_{2}$ afterglow and in the low pressure diffusion flame of the $\mathrm{CS}_{2}+\mathrm{O} / \mathrm{N}_{2}$ system is the $\mathrm{SO}_{2}$ in the $\widetilde{\mathrm{C}}$ state.

The next step to be investigated is to identify the $\mathrm{SO}_{2}{ }^{*}$ in the afterglow and in the flame with the vibronic levels of the $\widetilde{C}$ state. We measured the excess vibrational energy dependence of the magnetic quenching at $\mathrm{H}=12 \mathrm{kG}$ for twelve vibronic levels. The result is shown in Figure 9. As can be seen in the figure, the efficiency of the magnetic quenching increases rapidly with emitting level approaching to the predissociation threshold and it drops suddenly just above the threshold.

The efficiency of the magnetic quenching is dominated by the level density $(\rho)$ of the dark states which interact with the photoexcited singlet level. Both $\alpha$ of (7) and $\beta$ of (8) are proportional to $\rho .^{7}$ The above result implies that the density $\rho$ increases rapidly as the excited level approaches to the threshold. Above the threshold, the predissociation begins to occur and this sudden increase of the intrinsic 


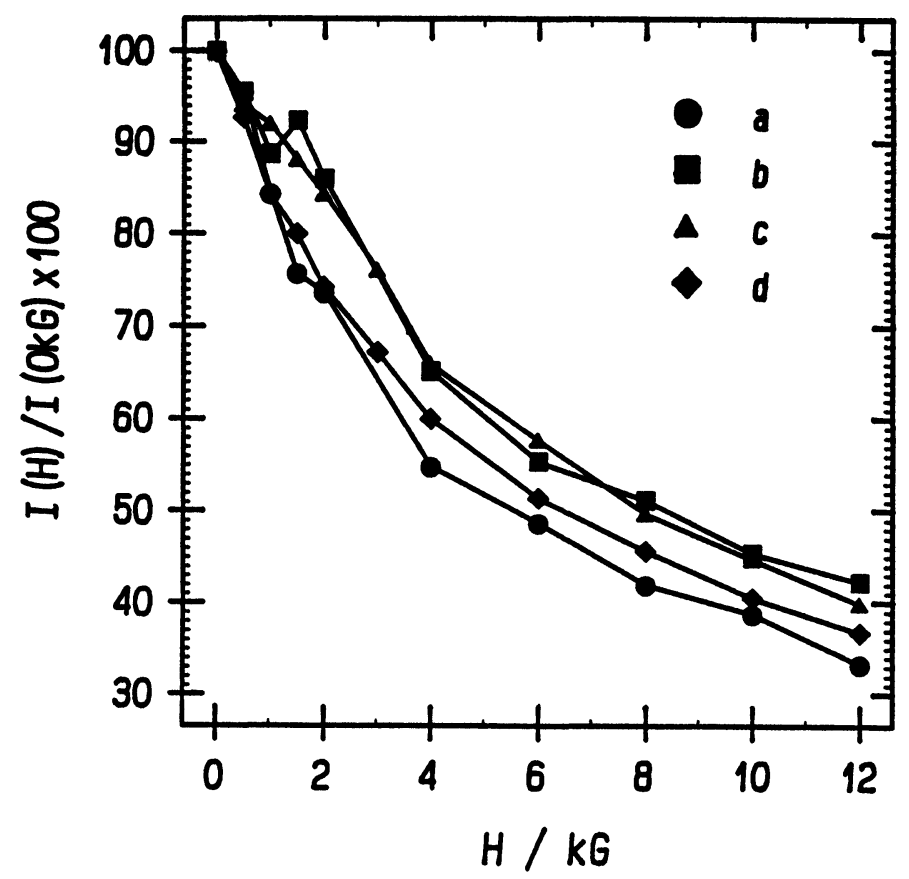

Figure 8 The field strength dependences of the magnetic quenchings observed for the peaks (a-d) indicated in Figure 7.

radiationless decay blurs the magnetic field induced change of $k_{n r}$. Therefore, as discussed in our previous paper, ${ }^{6}$ the necessary condition for the appearance of the magnetic field quenching of fluorescence has a close relevance to the level structure of the dark states near the predissociation threshold.

The key aim of the present study is concerned with the resultant vibronic levels of the reverse dissociation as denoted by the process (3). A comparison of the results shown in Figures 5 and 8 gives the suggestion that the $\mathrm{SO}_{2}{ }^{*}$ is the $\mathrm{SO}_{2}$ in the vibronic levels around the (112) level because of almost the same efficiencies of the magnetic quenchings at $\mathrm{H}=12 \mathrm{kG}$. A test to corroborate this suggestion would be the pressure dependence of the quenching, which is presented below.

\section{Collisional Effect on Magnetic Quenching of Fluorescence}

In order to simulate the pressure dependence of the magnetic quenching of chemiluminescence in the $\mathrm{CS}_{2}+\mathrm{O} / \mathrm{N}_{2}$ flame, we introduced the $\mathrm{N}_{2}$ gas up to 4 Torr into the chamber with keeping the partial pressure of $\mathrm{SO}_{2}$ at 0.024 Torr. Figure 10 shows the pressure dependences of the magnetic quenching efficiencies for five vibronic levels. At high pressures ( $>2$ Torr), the collisional quenching is the competitor with the magnetic quenching in the decay process and suppresses the magnetic quenching. On the other hand, at low pressures $(<1$ Torr), the collision assists the magnetic quenching in the efficiency, more or less, for all vibronic levels observed. 


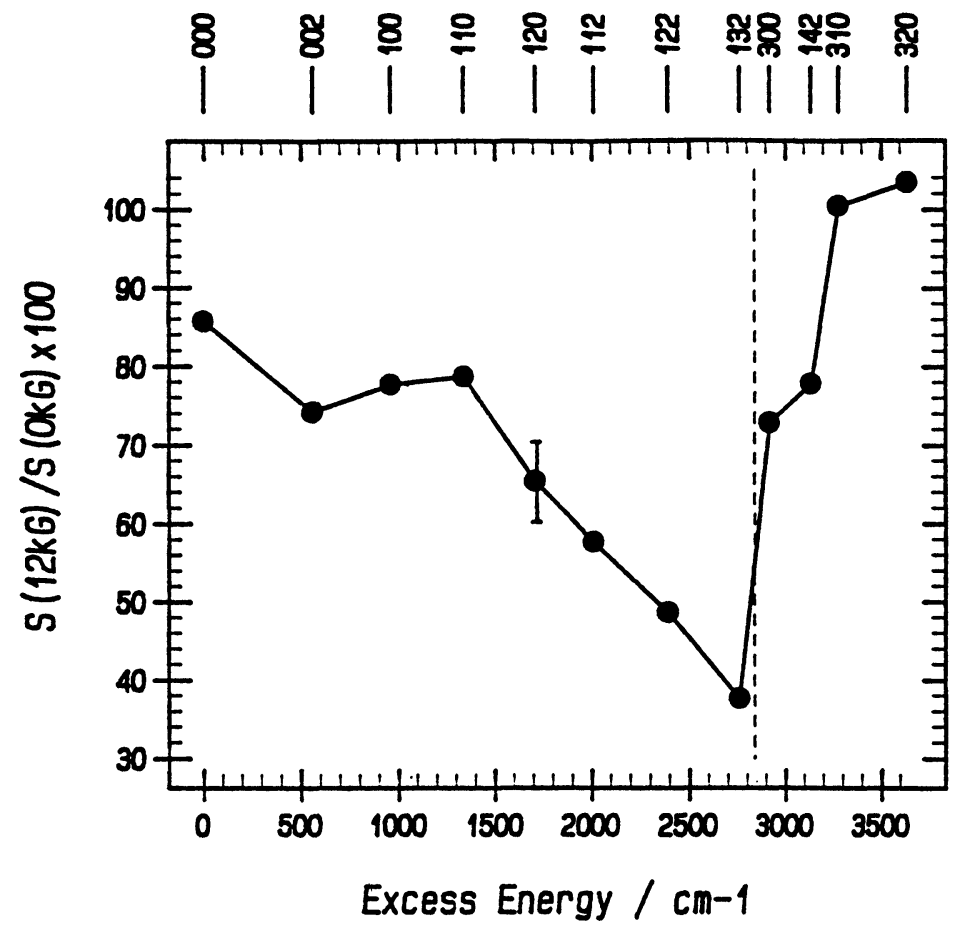

Figure 9 Excess energy dependence of the magnetic quenching at $\mathrm{H}=12 \mathrm{kG}$ observed for twelve vibronic bands of the $\mathrm{SO}_{2} \widetilde{\mathrm{C}}$ state. $\mathrm{S}(\mathrm{H})$ is the energy-integrated intensity over about $40 \mathrm{~cm}^{-1}$ around the head of each band at a magnetic field of $\mathrm{H}$. Assignment for each band taken from ref. 4 is indicated in the top. The vertical broken line represents the position of the predissociation threshold. An error bar typically represents three standard deviations for the data of at least six times measurements.

As the result, the efficiency of the magnetic quenching has its maximum at the medium pressure.

Similar but more definite collisional effect on the magnetic quenching of fluorescence was recently reported by Makarov and Khmelinski ${ }^{24}$ for the $\widetilde{A}{ }^{1} \mathrm{~A}_{2}$ and $\mathrm{B}$ ${ }^{1} \mathrm{~B}_{1}$ states of $\mathrm{SO}_{2}$, where the magnetic quenching is purely due to the collisional process. ${ }^{25,26}$ The magnetic quenching efficiencies for the $\mathrm{SO}_{2}$ self-collision have the maxima at very low pressures $(<0.1$ Torr). Because the lifetimes of these states are long (>100 $\mu \mathrm{s}),{ }^{25}$ the collision can sufficiently contribute to the nonradiative processes even at the low pressures. Furthermore, the efficiency of the quenching and the emission spectra from the $\widetilde{\mathrm{A}}$ and $\overline{\mathrm{B}}$ states are different from those shown in Figures 2 and 4 . These facts rule out the possibility that the $\mathrm{SO}_{2}{ }^{*}$ would be the $\mathrm{SO}_{2}$ in the high vibronic levels of the states.

Our results shown in Figure 10 indicate that the collision can somewhat contribute to the increase of $\rho$ through the collision-induced coupling within the dark levels. ${ }^{10,27}$ Note that the maximum of the magnetic quenching is the most definite for the levels at the mid excess energy, i.e. for the (112) and (122) levels. Especially the result for the (112) level is in qualitative agreement with that of chemiluminescence shown 


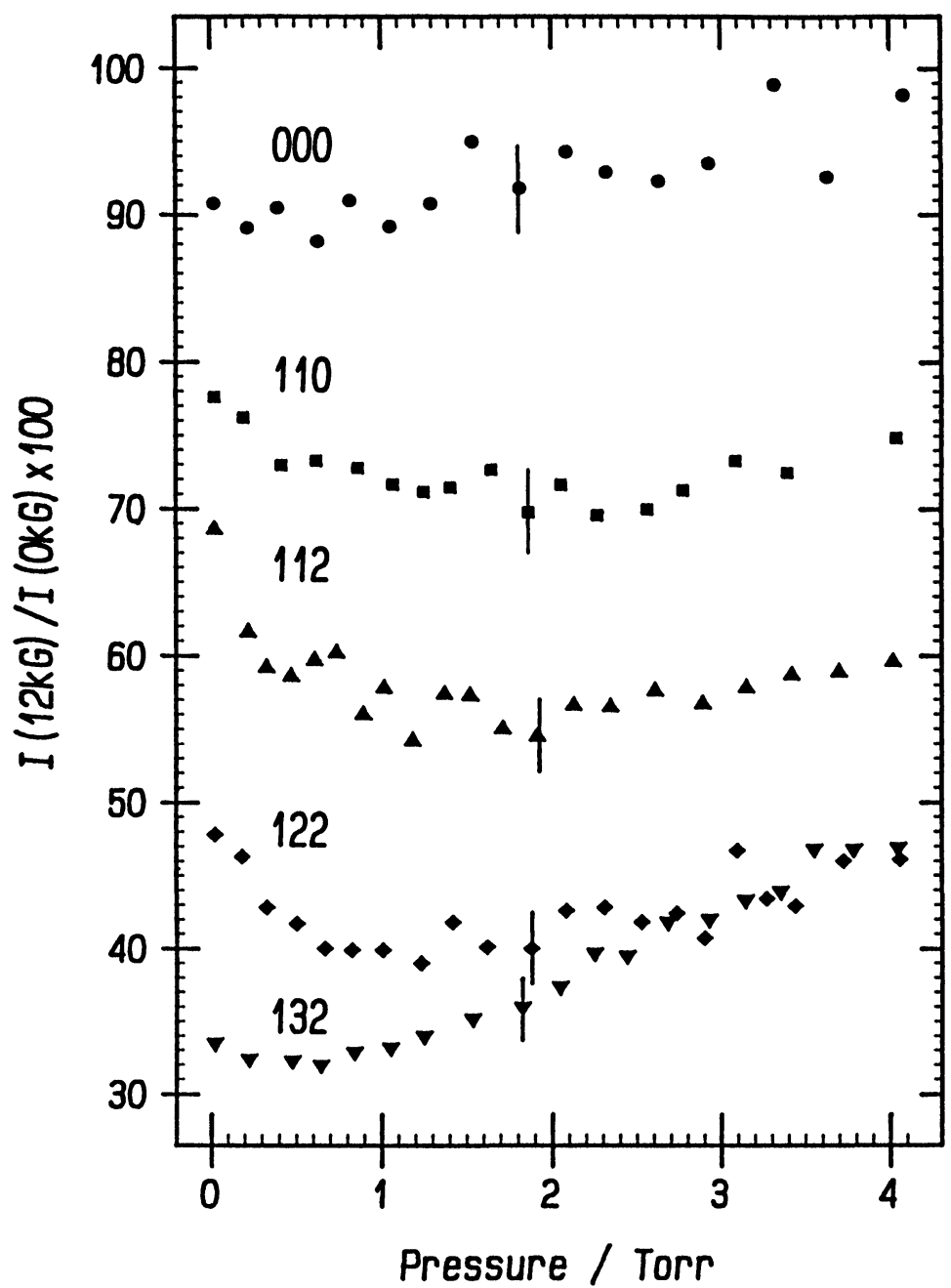

Figure 10 Pressure dependences of the magnetic quenchings at $\mathrm{H}=12 \mathrm{kG}$ observed for five vibronic bands of the $\widetilde{\mathrm{C}}$ state. The partial pressure of $\mathrm{SO}_{2}$ is kept at 0.024 Torr and total pressure is varied by the addition of $\mathrm{N}_{2}$. An error bar typically represents three standard deviations for the data of fourty times measurements.

in Figure $4 \mathrm{~b}$, both in the efficiency and in its pressure dependence. As can be seen in Figure 9, this energy region corresponds to that having the steepest energy dependence of the magnetic quenching efficiency.

Figure 11 shows the fluorescence decays from the (122) level in the absence and the presence of a magnetic field of $12 \mathrm{kG}$ at the pressures of $0.024,1.617$ and 3.878 Torr. As the pressure increases, the collisional quenching of fluorescence does certainly occur but the efficiency of the magnetic quenching shows less prominent pressure dependence than that of the collional quenching itself. This would be another evidence that the magnetic quenching is assisted in the efficiency by the collision. 


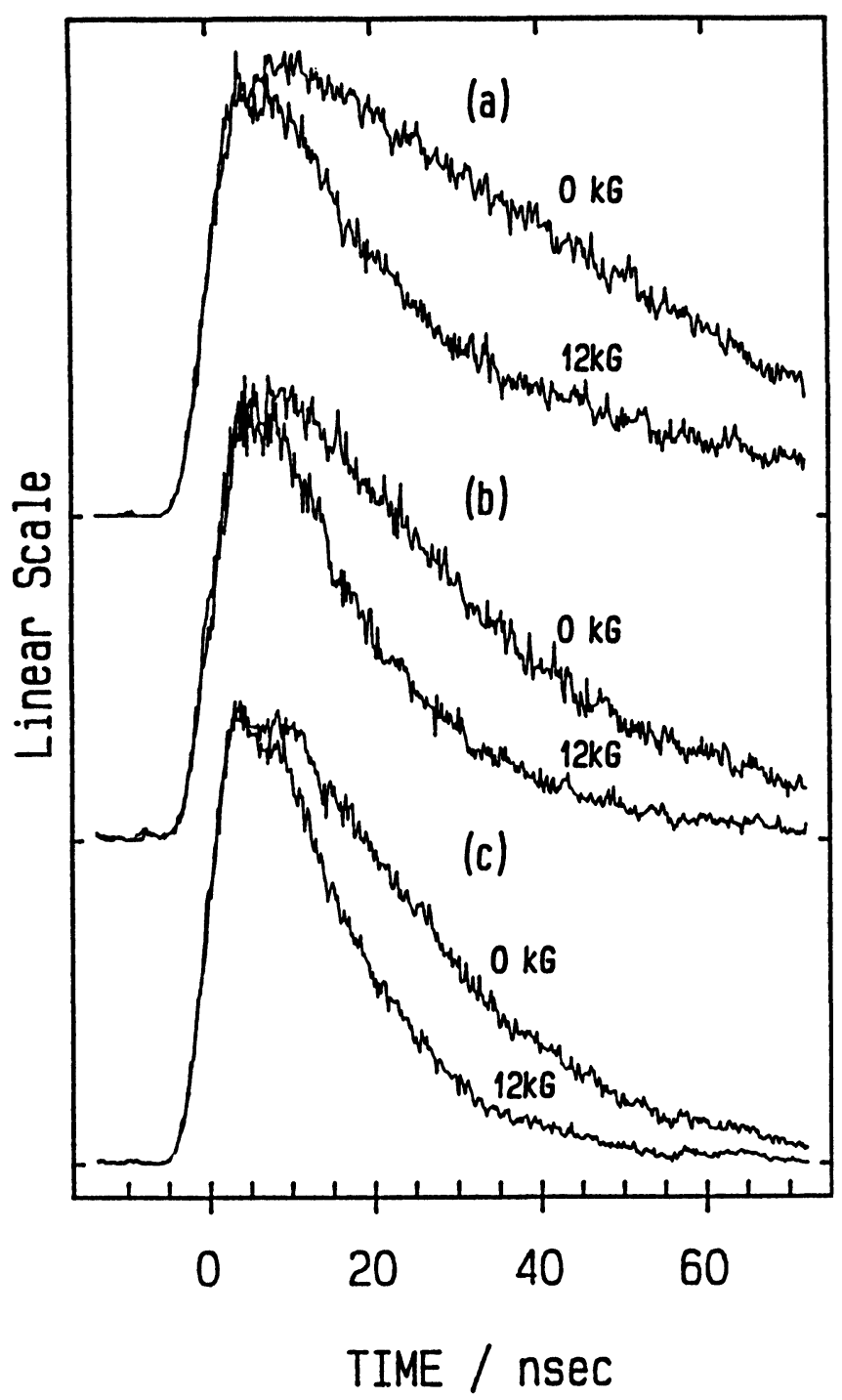

Figure 11 Fluorescence decays obtained by excitation of the strongest peak of the (122) band of the $\widetilde{\mathrm{C}}$ state under $\mathrm{H}=0$ and $12 \mathrm{kG}$ at the pressures of (a) 0.024 , (b) 1.617 and (c) 3.878 Torr. The pulse width of the laser is about $12 \mathrm{~ns}$ FWHM. The bandwidth of the detection is $150 \mathrm{MHz}$. Each decay is obtained by the accumulation of the data for 5200 laser shots.

\section{Single Vibronic Level Fluorescence Spectra}

The results presented in the previous two subsections indicate that the $\mathrm{SO}_{2} *$ in the low pressure diffusion flame of the $\mathrm{CS}_{2}+\mathrm{O} / \mathrm{N}_{2}$ system and in the $\mathrm{SO}_{2}$ afterglow is the $\mathrm{SO}_{2}$ in the vibronic levels around the (112) level of the $\widetilde{\mathrm{C}}$ state. This level is 
located at $\sim 800 \mathrm{~cm}^{-1}$ below the predissociation threshold (see Figure 9). The energy of $800 \mathrm{~cm}^{-1}$ would be reasonable in the amount for the energy which the third body $\left(\mathrm{N}_{2}\right)$ carries off from the reaction pair (SO and $\mathrm{O}$ ) during the three-body recombination of the process (3). The short lifetimes $(\sim 40 \mathrm{~ns})^{4,28}$ of the levels do not allow this small molecule to reach the equilibrium population by the collisions in the pressure range observed but do allow the collisional quenching to occur. Next, we consider how the chemiluminescence spectra are accounted for by this picture.

Figure 12 shows the dispersed fluorescence spectrum obtained by the excitation of the (132) band in a collision free condition. The emission spreads over more than $20000 \mathrm{~cm}^{-1}$ and the spectrum consists of very congested and complicated band structures. Ebata et $\mathrm{al}^{4}{ }^{4}$ and Yamanouchi et al ${ }^{29,30}$ observed similar spectra by the excitations of a few single vibronic levels (SVLs). They have shown that the complicated structure can be assigned to the vibrational structures of almost all possible combinations of the quantum numbers $\left(\mathrm{v}_{1}{ }^{\prime \prime}, \mathrm{v}_{2}{ }^{\prime \prime}, 2 \mathrm{v}_{3}{ }^{\prime \prime}\right)$.

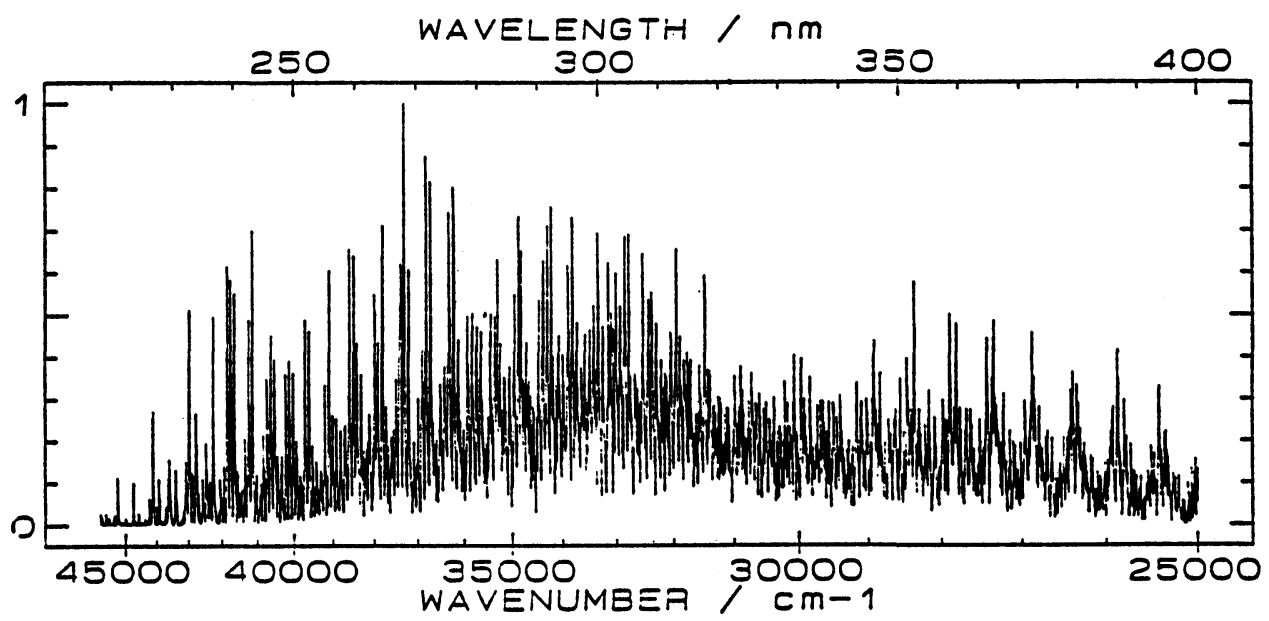

Figure 12 Dispersed fluorescence spectrum obtained by excitation of the (132) band (peak c in Figure 7 ) in a collision free condition. The spectral sensitivity of the detection system is not corrected.

Suppose that the three-body recombination process (3) produces the vibronic population consisting of the (probably more than ten) levels in the $\widetilde{C}$ state and that each vibronic level gives the complex SVL fluorescence spectrum as shown in Figure 12. Then the chemiluminescence spectra as shown in Figures 2 and 6 can be accounted to be the statistical overlap of many SVL fluorescence spectra. The structureless broad feature of chemiluminescence and its spectral distribution can be also rationalized by this picture. Note that the prepared levels by the recombination reaction are not necessarily optically accessible from the ground state. The feature of the magnetic quenching of chemiluminescence can be considered to be an average of those of the component vibronic levels in the $\widetilde{C}$ state. 


\section{CONCLUSIONS}

We have found that the chemiluminescence intensity in the low pressure diffusion flame of the $\mathrm{CS}_{2}+\mathrm{O} / \mathrm{N}_{2}$ system is considerably affected by external magnetic fields. The emitter in the flame was found to be the same as the main emitter in the $\mathrm{SO}_{2}$ afterglow. We have characterized this MFE by the measurements of its field strength dependence, collisional effect and spectral distribution. The major emitter was considered to be the $\mathrm{SO}_{2}$ in the $\widetilde{C}{ }^{1} \mathrm{~B}_{2}$ state. We have measured the MFE on the fluorescence from the vibronic levels in the $\widetilde{C}$ state. It was found that external magnetic fields quench fluorescence remarkably from the levels just below the predissociation threshold. From the observed vibrational-level, field-strength and pressure dependences of the magnetic quenchings, we could specify the major emitter of chemiluminescence to be the $\mathrm{SO}_{2}$ molecule in the levels around the (112) one of the $\widetilde{C}$ state. This was also supported by the good correspondence between the features of the chemiluminescence spectra and the SVL fluorescence spectra emitted from these vibronic levels.

The minor emission in the flame showed the magnetic enhancement in the intensity. The origin of the emission is not clear at present.

We have presented an example in this paper which demonstrates that the MFE on luminescence can give the important informations on its emitter itself as well as on the dynamic behavior in the excited state..$^{20}$

\section{Acknowledgements}

The author thanks Prof. Hisaharu Hayashi for his useful suggestions. This work was supported by Special Research Promotion Fund (1991) at RIKEN and by a Grantin-Aid for Scientific Research on Priority Area "Molecular Magnetism" (No. 228/04242102) from the Ministry of Education, Science and Culture, Japan.

\section{References}

1. A. G. Gaydon. Proc. Roy. Soc., A146, 901 (1934).

2. L. Herman, J. Akriche and H. Grenat. J. Quant. Spectry. Radiative Transfer., 2, 215 (1962).

3. H. Okabe. J. Am. Chem. Soc., 93, 7095 (1971).

4. T. Ebata, O. Nakazawa and M. Ito. Chem. Phys. Letters., 143, 31 (1988).

5. C. J. Halstead and B. A. Thrush. Proc. Roy. Soc., A295, 363 (1966).

6. H. Abe and H. Hayashi. Chem. Phys. Letters., 187, 227 (1991).

7. P. R. Stannard. J. Chem. Phys., 68, 3932 (1978).

8. A. Matsuzaki and S. Nagakura. Helv. Chim. Acta., 61, 675 (1978).

9. H. Abe and H. Hayashi. Chem. Phys., 162, 225 (1992).

10. T. Yoshidome, H. Abe and H. Hayashi. J. Phys. Chem., 96, 34 (1992).

11. G. B. Kistiakowsky and G. G. Volpi. J. Chem. Phys., 27, 1141 (1957).

12. F. Kaufman and J. Kelso. J. Chem. Phys., 27, 1209 (1957).

13. H. Abe and H. Hayashi. Chem. Phys. Letters., 206, 337 (1993).

14. W. F. Cooper and J. F. Hershberger. J. Phys. Chem., 96, 5405 (1992).

15. R. W. B. Pearse and A. G. Gaydon, The Identification of Molecular Spectra (Chapman and Hall, London, 1984), 4th ed., pp. 298.

16. R. Colin, Can. J. Phys., 47, 979 (1969). 
17. J. Duchesne and B. Rosen. J. Chem. Phys., 15, 631 (1947).

18. J. C. D. Brand and K. Srikameswaran. Chem. Phys. Letters., 15, 130 (1972).

19. J. C. D. Brand, P. H. Chiu, A. R. Hoy and H. D. Bist. J. Mol. Spectry., 60, 43 (1976).

20. for a review; H. Hayashi, Photochemistry and Photophysics, Vol. 1, ed. J. F. Rabek (CRC Press, Boca Raton, 1990), pp. 59.

21. H. Abe, H. Hayashi, T. Imamura and S. Nagakura. Chem. Phys., 137, 297 (1989).

22. N. Ohta and T. Takemura. J. Chem. Phys., 95, 7119 (1991).

23. N. Ohta and T. Takemura. J. Chem. Phys., 95, 7133 (1991).

24. V. I. Makarov and I. V. Khmelinski. J. Photochem. Photobiol., A69, 7 (1992).

25. V. I. Makarov, N. L. Lavrik and N. M. Bazhin. Chem. Phys., 72, 213 (1982).

26. V. I. Makarov, N. L. Lavrik, G. I. Skubnevskaya and N. M. Bazhin. Opt. i Spectroskopiya., 50, 154 (1981).

27. K. F. Freed. Adv. Chem. Phys., 47, 291 (1981).

28. M. H. Hui and S. A. Rice. Chem. Phys. Letters., 17, 474 (1972).

29. K. Yamanouchi, H. Yamada and S. Tsuchiya. J. Chem. Phys., 88, 4664 (1988).

30. K. Yamanouchi, S. Takeuchi and S. Tsuchiya. Prog. Theor. Phys. Sup., 98, 420 (1989). 